\title{
Multichannel Receivers for OFDM and TDMA in Mobile Communications
}

\author{
Meritxell Lamarca, Gregori Vázquez \\ Dept. of Signal Theory and Communications, Polytechnic University of Catalonia \\ c/Gran Capità, 08034-Barcelona (Spain); email: [xell,gregori]@gps.tsc.upc.es
}

\begin{abstract}
This paper addresses the use of multichannel receivers for blind equalization in TDMA under frequency selective channels and OFDM systems in frequency flat fading channels. A new criteria is proposed for blind equalization of finite length mobile channels.
\end{abstract}

\section{Introduction}

Mobile communications operate in a very hostile environment due to multipath propagation and vehicle displacement. Depending on the transmission rate and vehicle speed, either frequency-selectivity or Doppler spectrum spreading becomes the major concern. However, in both cases, the receiver must be able to track and compensate channel distortion.

In this paper, we propose a new spatio-temporal linear equalization technique which can be applied to block transmission schemes (e.g..TDMA) operating frequency selective channels and also to OFDM modulation subject to multiplicative distortion (frequency flat fading or $\mathrm{F}^{3}$ ).

The suggested approach is blind and needs of the use of diversity receivers which will allow for the application of multichannel formulation. In the case of frequency selective channels, this diversity can be achieved either by means of oversampling the received signal or using spatial diversity, whereas it will be shown that in the case of OFDM modulation in multiplicative channels spatial diversity is necessary.

The proposed method only exploits the fact that the transmitted signal is the same in all the channels, but makes no assumptions on its value. The algorithm can be classified as a blind deterministic criteria and so, its performance and its constraints are similar to those of other methods of the same type ([1]), that is, it obtains good results for relatively short sets of data, it assumes the channel is FIR with known length and its derivation does not take into account the additive noise. As we will see, the impact of the noise will be considered in the optimization procedure of the algorithm, later on.

\section{Problem formulation}

For convolutive channels in TDMA and $\mathrm{F}^{3}$ channels in OFDM, it is possible to express the channel distortion

This work has been partially supported by the Spanish Research Council (TIC-95-1022-C05-01, TIC-96-0500-C10-01) and by CIRIT/ Generalitat de Catalunya (SGR0096) as a linear convolution. Assuming D-th order diversity is available, for each of the receiving channels we have:

$$
Y_{i}(z)=T(z) \cdot C_{i}(z)+W_{i}(z) \quad i=1, \ldots D
$$

being $T(z), Y_{i}(z), C_{i}(z)$ and $W_{i}(z)$, the $z$-transforms of the transmitted and received signals, the channel response and noise term in the $i$-th channel respectively. Notice that if z-transforms are regarded as polynomials, it turns out that in the noiseless case (assuming $\mathrm{C}_{\mathrm{i}}(\mathrm{z})$ coprime):

$$
T(z)=\operatorname{g.c.d} .\left\{Y_{1}(z), Y_{2}(z), \ldots, Y_{D}(z)\right\}
$$

where g.c.d $\{\cdot\}$ stands for the greatest common divisor. The proposed equalizer is based on this fact. It will try to estimate the greatest common divisor of all $\mathrm{Y}_{\mathbf{i}}(\mathrm{z})$, knowing that it is equivalent to the estimation of the transmitted signal. Of course, in order to make use of this property, the receiver must be able to observe the complete signals $Y_{i}(z)$. This is the main difference with respect to other blind algorithms which have been developed in the past. The equalizer will be developed under the assumption that the receiver observes the complete convolution output. Although $\mathrm{Y}_{\mathrm{i}}(\mathrm{z})$ is not available if continuous transmission schemes are used, this is not a major constraint because block transmission is the most common scherne in mobile communications (for both TDD and FDD). Next, two different environments are shown where the present approach can be applied.

Case a. Block transmission systems operating in convolutive channels.

The proposed algorithm can be applied here if after each frame is transmitted, a guard-time is introduced. If the guard-time is longer than channel impulse response duration then inter-frame interference disappears and $\mathrm{Y}_{\mathrm{i}}(\mathrm{z})$ can be fully observed. Notice that this case includes both single carrier and OFDM modulation systems, although in the case of OFDM, the usual cyclic prefix extension ([2]) should be replaced by the already mentioned guardtime. In the single carrier case, the diversity can be achieved either by means of oversampling or by means of spatial diversity.

Case b. OFDM in $F^{3}$ channels.

Although in this case the distortion becomes multiplicative, it can be compensated by means of 
multichannel filtering, as shown next. Fig.1 summarizes the scenario we will deal with (only those stages of the OFDM transmission system relevant to our analysis are shown). In [3] the authors recognized that because of the IDFT/DFT performed at both ends of the channel, the multiplicative distortion behaved in the transformed domain as a convolutive channel. However, the convolution performed was circular $(\mathrm{CC})(\otimes)$ rather than linear (LC):

$$
Y_{i}[k]=T[k] \otimes C_{i}[k]+W_{i}[k]
$$

Unfortunately, equation (1) does not hold for circular convolution ([4]) and so the channel cannot be equalized by means of (2). In spite of that, it can be seen that oversampling the received signals $y_{i}(t)$ is equivalent to zero padding the transformed domain sequences $T[z]$, $\mathrm{C}_{1}[\mathrm{z}]$ and $\mathrm{C}_{2}[\mathrm{z}]$ as long as there was no aliasing introduced when they were sampled at the symbol rate. Moreover, as a result of the zero padding the $\mathrm{CC}$ will operate as a LC of the not-oversampled sequences:

$$
Y_{i}[k]=T_{Z P}[k] \otimes C_{i_{Z P}}[k]+W_{i_{P P}}[k]=T[k] * C_{i}[k]+W_{i_{Z P}}[k]
$$

In other words, in OFDM the CC can be turned into a LC $\left({ }^{*}\right)$ at the expense of doubling the sampling rate. Notice that in this case oversampling is necessary, but it does not provide the desired diversity and so, more than one antenna will be required.

As will be shown next, the equalizer will be developed under the hypothesis of having FIR transmission channels. In the case of OFDM in $\mathrm{F}^{3}$ environments, the channel will be slowly varying and therefore it can be regarded as a low-pass signal. However, its description by means of only low frequency components $(\mathrm{C}[\mathrm{k}]$ of finite length) is approximated, and the equalizer hypothesis will not be completely fulfilled in this case. This will be an obstacle for equalizer performance, but simulations will show that the proposed algorithm is robust and also works for the case of OFDM in $\mathrm{F}^{3}$ channels.

\section{Algorithm basis}

In this section we will show how equation (2) can be used to blindly estimate the equalizer coefficients and in section 4 we provide an algorithm based o this idea. The proposed algorithm is based on a generalization of the Bezout equation ([5],[6]).

Property: Given a set of $D$ polynomials $\mathrm{A}_{\mathbf{i}}(\mathrm{z})$, then, the equation:

$$
\sum_{i=1}^{D} A_{i}(z) \cdot a_{i}(z)=1
$$

has a solution in $\mathrm{a}_{\mathrm{i}}(\mathrm{z})$ iff $\mathrm{A}_{\mathrm{i}}(\mathrm{z})$ are coprime. Furthermore, assuming $A_{i}(z)$ have all degree $L-1$, then the polynomials $a_{\mathbf{i}}(z)$ have degree $M \geq(L-1) /(D-1)$.

The relationship of equation (5) with multichannel equalization and perfect reconstruction filter banks has been acknowledged in the literature ([7]) and will become evident once we will have formulated the equalization problem mathematically. Figure 2 shows a block diagram of the equivalent transmission channel and linear equalizer for the multichannel formulation. In this figure, $E_{i}(z)$ stands for the $i$-th channel equalizer response and $Q(z)$ for the equalize output.

As shown, we will use FIR filters to equalize FIR channels up to a multiplicative constant. As was noticed in the literature too, this property of multichannel approaches means that the zero-forcing equalizer does not necessarily have to emphasize noise, although in any case it will not perform in terms of noise as well as the MMSE equalizer. Besides, it is worth mentioning that in OFDM in multiplicative channels an FIR channel can be perfectly equalized by means of another FIR filter.

The output of the equalizer of this structure follows the equation

$$
Q(z)=\sum_{i=1}^{D} Y_{i}(z) \cdot E_{i}(z)=T(z) \cdot \sum_{i=1}^{D} C_{i}(z) \cdot E_{i}(z)
$$

This equation states that $\mathrm{Q}(\mathrm{z})$ will always be a multiple of $\mathrm{T}(\mathrm{z}) . \mathrm{Q}[\mathrm{k}]$ will be the result of convolving the transmitted data $T[k]$ with the equivalent channel-plusequalizer filter $\mathrm{H}(\mathrm{z})$

$$
Q(z)=T(z) \cdot H(z) \quad ; \quad H(z)=\sum_{i=1}^{D} C_{i}(z) \cdot E_{i}(z)
$$

Furthermore, notice that in order to achieve perfect equalization (in the zero-forcing sense), i.e. $Q(z)=T(z)$, the following equation must be verified:

$$
H(z)=\sum_{i=1}^{D} C_{i}(z) \cdot E_{i}(z)=1
$$

Notice that $\operatorname{deg}\{\mathrm{T}(\mathrm{z}) \mathrm{H}(\mathrm{z})\} \geq \operatorname{deg}\{\mathrm{T}(\mathrm{z})\}$, with equality only when $\mathrm{H}(\mathrm{z})=1$ (up to a multiplicative constant). Therefore, asking for $\mathrm{Q}(\mathrm{z})$ of minimum degree tantamounts to asking for $Q(z)=T(z)$. This is the condition over which the blind algorithm will be built. The equalizer will be designed to yield an estmate $Q(z)$ of minimum length and this will lead it to the ISI-free solution.

Equation (8) is in fact a particular case of (5), where $A_{i}(z)=C_{i}(z)$ and $a_{i}(z)=E_{i}(z)$. Therefore, the property above tells us when this procedure will be feasible. It will be able to achieve an ISI-free output if the channel responses $\mathrm{C}_{\mathrm{i}}(\mathrm{z})$ are coprime and if we let $\mathrm{E}_{\mathrm{i}}(\mathrm{z})$ to have enough coefficients. 
The coprimeness condition is well known in the literature and applies to all blind channel/equalizer identification techniques based on second-order statistics and deterministic approaches ([8],[1]). Notice that if the channels were not coprime, the g.c.d $\left\{\mathrm{Y}_{\mathrm{i}}(\mathrm{z})\right\}$ would not yield $T(z)$, because it would rather include the common factors of all channels too.

The fact that equation (8) has infinite solutions means that the zero-forcing solution is not unique, being the difference among all possible solutions their behavior in front of the noise ([9]). Two particular cases are specially interesting: $D=2$ and $D=L$. In the dual-diversity case $(D=2)$, we can find a closed form for all zero-forcing solutions ([10]):

$$
\begin{aligned}
& E_{1}(z)=E_{1}^{o}(z)-C_{2}(z) \cdot F(z) \\
& E_{2}(z)=E_{2}^{o}(z)+C_{1}(z) \cdot F(z)
\end{aligned}
$$

being $E_{1}{ }^{\circ}(z)$ and $E_{2}{ }^{\circ}(z)$ the only solution of length $\dot{M}=\mathrm{L}-1$ (any other solution will have at least length $\mathrm{L}$ ) and $F(z)$ an arbitrary factor which provides the infinite available solutions. The fact that in the two channel case, a unique solution exists for $\mathrm{M}=\mathrm{L}-1$ is well known ([7]).

In the extreme case of $D=L$, it turns out that $M \geq 1$. In the spatial diversity case this means that the ISI dispersive channel can be perfectly equalized by means of spatial filtering only, a fact which was also previously reported [11].

\section{Algorithm formulation}

Although the procedure can be applied to any number of channels $D$, fo the sake of simpliciy the algorithm will be developed for the dual diversity receiver $(D=2)$. In this case, any zero-forcing solution for $E_{1}(z)$ and $E_{2}(z)$ will satisfy

$$
T(z)=Y_{1}(z) E_{1}(z)+Y_{2}(z) E_{2}(z)
$$

This equation can be written in matrix form as in equations (11) and (12). Notice that the formulation will be different for the two cases considered in section 2 .

Case a. Block transmission in Convolutional channels

In this case eq. (10) yields a Sylvester matrix

$$
\left[\begin{array}{cccccccc}
y_{11} & 0 & \ldots & 0 & y_{21} & 0 & \ldots & 0 \\
y_{12} & y_{11} & \ldots & \ldots & y_{22} & y_{21} & \ldots & \ldots \\
y_{13} & y_{12} & \ldots & 0 & y_{23} & y_{22} & \ldots & 0 \\
\ldots & y_{13} & \ldots & 0 & \ldots & y_{23} & \ldots & 0 \\
y_{1 d} & \ldots & \ldots & y_{11} & y_{2 d} & \ldots & \ldots & y_{21} \\
0 & y_{1 d} & \ldots & y_{12} & 0 & y_{2 d} & \ldots & y_{22} \\
\ldots & 0 & \ldots & y_{13} & \ldots & 0 & \ldots & y_{23} \\
0 & \ldots & \ldots & \ldots & 0 & \ldots & \ldots & \ldots \\
0 & 0 & \ldots & y_{1 d} & 0 & 0 & \ldots & y_{2 d}
\end{array}\right]\left[\begin{array}{c}
e_{11} \\
e_{12} \\
\vdots \\
e_{1, M-1} \\
e_{21} \\
e_{22} \\
\vdots \\
e_{2, M-1}
\end{array}\right]=\left[\begin{array}{c}
t_{1} \\
t_{2} \\
\vdots \\
t_{N} \\
0 \\
0 \\
\vdots \\
0 \\
0
\end{array}\right]
$$

where $d$ is the duration of the transmitted frame at the output of the channel, that is, if the length of the transmitted frame is denoted by $\mathrm{N}$ then $\mathrm{d}=\mathrm{N}+\mathrm{L}-\mathrm{I}$.

\section{Case b. OFDM in a multiplicative channel}

As was mentioned in section 2, the description of the channel response in the transform domain $\mathrm{C}[\mathrm{k}]$ as a short duration sequence is only approximated. Therefore, even if the received signal is oversmpled, it will still be the result of a circular convolution. To take into account this fact, in this case we have modified matrix formulation of the equalizer. the new equations take into account the circular nature of the DFT and in the case of oversampling by a factor of 2 they are

$\left[\begin{array}{cccccccc}y_{11} & y_{1,2 N} & \ldots & y_{1,2 N-L-1} & y_{21} & y_{2,2 N} & \ldots & y_{2,2 N-L-1} \\ y_{12} & y_{11} & \ldots & \vdots & y_{22} & y_{21} & \ldots & \vdots \\ y_{13} & y_{12} & \ldots & y_{11} & y_{23} & y_{32} & \ldots & y_{21} \\ \vdots & \vdots & \ldots & \vdots & \vdots & \vdots & \ldots & \vdots \\ y_{1,2 N} & y_{1,2 N-1} & \ldots & y_{1,2 N-L-2} & y_{2,2 N} & y_{2,2 N-1} & \ldots & y_{2,2 N-L-2}\end{array}\right]\left[\begin{array}{c}e_{11} \\ e_{12} \\ \vdots \\ e_{1, L-1} \\ e_{21} \\ e_{22} \\ \vdots \\ e_{2, L-1}\end{array}\right]=\left[\begin{array}{c}t_{1} \\ t_{2} \\ \vdots \\ t_{N} \\ 0 \\ 0 \\ \vdots \\ 0\end{array}\right](12)$

Both, eq. (11) and (12) can be represented in as follows:

$$
\left[\begin{array}{l}
\underline{\underline{Y}} t \\
\underline{\underline{Y}}_{0}
\end{array}\right]\left[\begin{array}{l}
\underline{E}_{1} \\
\underline{E}_{2}
\end{array}\right]=\left[\begin{array}{l}
\underline{T} \\
\underline{\underline{a}}
\end{array}\right] \text { or }\left[\begin{array}{l}
\underline{\underline{Y}}_{t} \\
\underline{\underline{Y}}_{0}
\end{array}\right] \underline{\underline{E}}=\left[\begin{array}{l}
\underline{T} \\
\underline{0}
\end{array}\right]
$$

where the set of equations has been grouped in two sets: $\left\{\underline{\underline{Y}}_{\mathrm{t}}, \mathrm{I}\right\}$ and $\left\{\underline{\underline{Y}}_{\mathrm{o}}, \underline{0}\right\}$. Notice that the second set of equations only depends on the chanhel output. Thus, it is possible to estimate the channel equalizer by considering the following set of linear equations:

$$
\underline{Y}_{0} \underline{E}=\underline{0}
$$

Equation (14) tantamounts to requiring the equalizer output to have minimum length, as described in section 3. Furthermore, once an estimate of the channel equalizer has been obtained, it is possible to recover the transmitted data:

$$
\underline{\underline{T}}=\underline{\underline{Y}}_{t} \underline{\underline{E}}
$$

It is important to remark that the presence of the noise in the channel implies that equation (14) can not be longer satisfied. If the channel is perfectly zero-forced by the equalizer $\underline{E}$, then, the residue in equation (14) will be due only to the noise at the output of the equalizer. Thus, if we want a channel equalization taking into account the noise level and spectral distribution for a better symbol decisions, we can minimize the following cost function:

$$
\min _{\underline{E}} \underline{E}^{H} \underline{\underline{Y}}_{0}^{H} \underline{\underline{Y}}_{0} \underline{E}
$$


with any non-trivial constraint. The best performance of this criteria was found when taking the noise sub-space of matrix $\underline{\underline{Y}}_{0}$ and applying the minimum-norm algorithm. Other more simple non-trivial constrains have been also considered with some performance degradation.

\section{Simulations}

The simulations in fig. 3 illustrate the behavior of the algorithm in the more demanding case of OFDM and multiplicative distortion (the convolutional channel case performs better). Fig.3a-3f show the results obtained for a particular realization of the mobile channel with the following parameters: OFDM frame of 128 QPSK symbols, $\mathrm{EbNo}=22 \mathrm{~dB}$, Rayleigh fading channel corresponding to $f_{\text {carrier }}=1 \mathrm{GHz}$, vehicle speed: $100 \mathrm{Km} / \mathrm{h}$, transmission rate $50 \mathrm{~Kb} / \mathrm{s}$, oversampling factor: 2 , equalizer length $\mathrm{M}=5$, noise subspace dimension: 2 . Figure $3 \mathrm{~g}$ illustrates the performance averaged over 500 channel realizations with the same parameters except for the EbNo, which was given two values: 22 and $32 \mathrm{~dB}$. It shows the $\%$ of transmitted frames for which the received $\mathrm{EbNo}$ in $\mathrm{dB}$ was below a specified threshold. Notice that because of the random behavior of the channel sometimes the output EbNo is higher than the input one.

\section{References}

[1] G.Xu, et al, 'A Least-Squares Approach to Blind Channel Identification', IEEE Trans. on Signal Processing, vol.43, No.12, pp.2982,2993

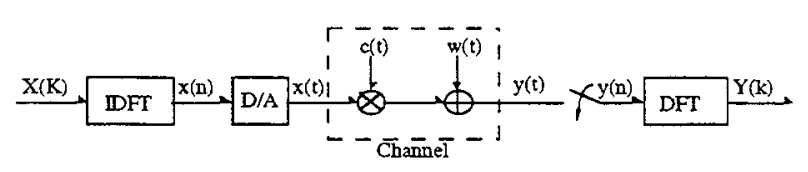

Fig.1. Simplified model of an OFDM transmission system for a frequency flat fading channel.

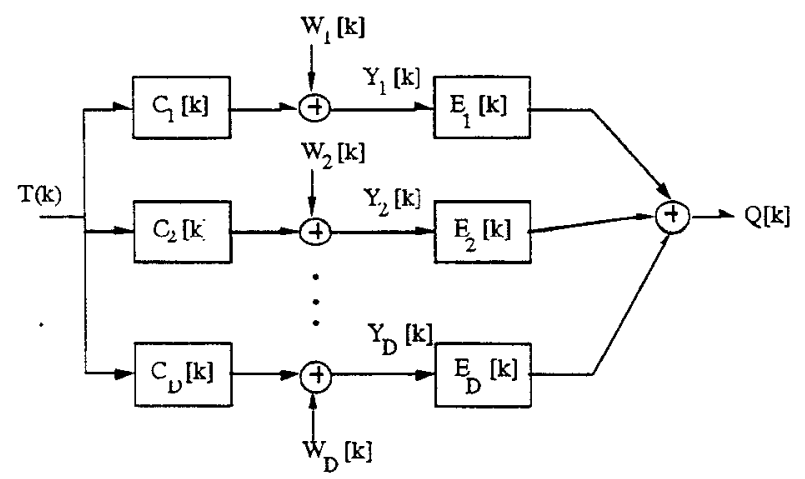

Fig.2. Model of the D-th ordr diversity receiver and the channel equalization stage.
[2] W.Zou, Y.Wu, 'COFDM: An Overview', IEEE Trans. on Broadcasting, vol.41, No.1, March 1995

[3] J.Ahn, H.S.Lee, 'Frequency domain equalization of OFDM signals over frequency nonselective Rayleigh fading channels', Electronic Letters, Vol.19 No.16, pp.1476-1477

[4] M.Lamarca, G.Vázquez, 'Channel estimation for transform modulations in mobile communications', Proc. of EUSIPCO'96, Trieste (Italy), pp.1977-1980

[5] T.Kailath, Linear Systems, Prentice-Hall, 1980

[6] J.H.McClellan and C.M.Rader, Number Theory in Digital Signal Processing, Prentice Hall, 1979

[7] D.T.M.Slock, 'Blind fractionally spaced equalization, perfect reconstruction filter banks and multichannel linear prediction', Proc. of ICASSP'95, Adelaida (Australia), pp.IV.585-IV.588

[8] Y.Li, Z.Ding, 'Blind Channel Identification based on second order cyclostationary statistics', Proc. of ICASSP'93, Minneapolis(USA), pp. IV.81-IV.84

[9] G.Giannakis,S.D.Halford, 'Blind fractionally-spaced equalization of noisy FIR channels: Adaptive and optimal solutions', Proc. of ICASSP'95, Detroit (USA), pp.1972-1975

[10] C.W.Kok, T.Q.Nguyen, 'Chinese Remainder Theorem: Recent trends and new results in filter banks design', Proc. of EUSIPCO'96, Trieste (Italy), pp.755-758

[11] S.Mayrargue, 'Spatial equalization of a radio-mobile channel without beamforming using the Constant Modulus Algorithm (CMA)', Proc. of ICASSP'93, Minneapolis(USA), pp. III.344-III.347
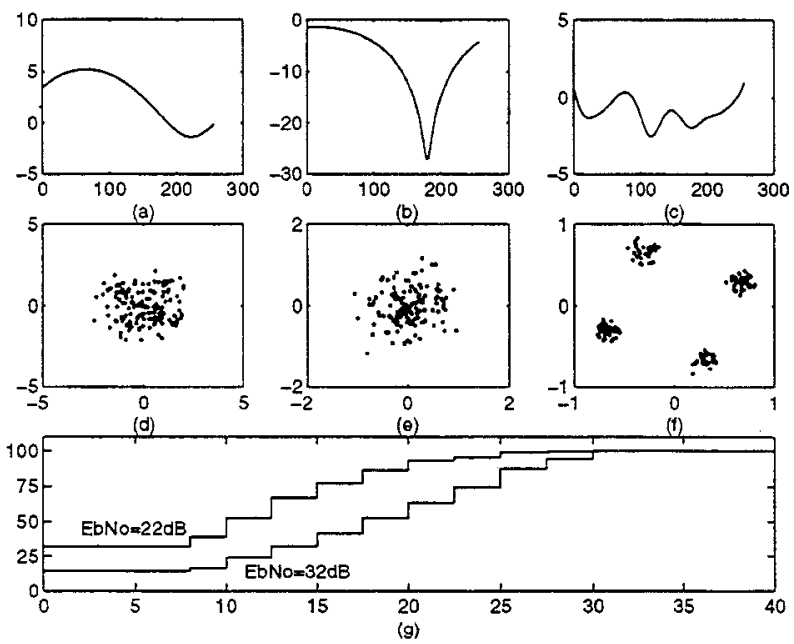

Fig.3. Algorithm simulation. (a)-(c) Multiplicative response of $\mathrm{C}_{1}(\mathrm{z}), \mathrm{C}_{2}(\mathrm{z})$ and $\mathrm{H}(\mathrm{z})$; (d)-(f) Constellations for $\mathrm{Y}_{1}[\mathrm{k}]$, $\mathrm{Y}_{2}[\mathrm{k}]$, and $\mathrm{Q}[\mathrm{k}] ;(\mathrm{g})$ (see section 5 ) 